\title{
Sealing Effects on the Storage Stability of the Cyanide Antidotal Candidate, Dimethyl Trisulfide
}

\author{
Lóránd Kiss $^{1}{ }^{(1)} \cdot$ Anna Duke $^{1}\left(\right.$ Kristof Kovacs $^{1}{ }^{1} \cdot$ Tibor Barcza $^{1} \cdot$ \\ Márton Kiss ${ }^{1}\left(\mathbb{D} \cdot\right.$ Ilona Petrikovics $^{1}$ (D) David E. Thompson ${ }^{1}$ (D)
}

Published online: 6 December 2017

(c) The Author(s) 2017. This article is an open access publication

\begin{abstract}
Background Dimethyl trisulfide (DMTS) is a highly lipidsoluble cyanide $(\mathrm{CN})$ antidote candidate molecule. In prior studies with various US FDA-approved co-solvents, surfactants, and their combinations, aqueous solutions containing $15 \%$ polysorbate 80 (Poly80) were found to effectively solubilize DMTS in formulations for intramuscular administration. However, DMTS formulated in $15 \%$ aqueous Poly80 solutions showed gradual losses over time when stored in vials with septum-based seals.

Objective The present study tested whether storing DMTS formulations in hermetically sealed glass ampules could mitigate storage losses.

Methods Samples consisted of 1-mL aliquots of a $50 \mathrm{mg} /$ $\mathrm{ml}$ stock solution of DMTS in 15\% aqueous Poly80. The control samples were stored using a vial-within-a-vial system - the inner and outer vials were sealed respectively, with a snap cap, and with a crimped septum. The hermetically sealed test samples were stored in fire-sealed glass ampules. The DMTS content was measured by HPLC-UV analysis at specific time points over a 100-day period.

Results While the control samples exhibited systematic DMTS losses, no DMTS losses were observed from the test samples stored in hermetically sealed glass ampules over the 100-day testing period.

Conclusion DMTS formulated in 15\% aqueous Poly80 solution has excellent stability when stored in fire-sealed glass ampules and thus has the potential to be effectively stored as an intramuscular $\mathrm{CN}$ countermeasure for mass casualty scenarios.
\end{abstract}

David E. Thompson

david.thompson@shsu.edu

1 Department of Chemistry, Sam Houston State University, Huntsville, TX 77341, USA

\section{Key Points}

An improved storage approach is reported for the promising cyanide antidote dimethyl trisulfide (DMTS).

DMTS exhibited no measurable loss over the 100-day study period when stored in hermetically sealed glass ampules.

\section{Introduction}

Cyanide $(\mathrm{CN})$ causes toxicity by interfering with cellular respiration. In humans, the endogenous sulfurtransferase enzyme rhodanese ( $\mathrm{Rh}$ ) detoxifies $\mathrm{CN}$ by converting it to the less toxic thiocyanate $[1,2]$; however, this detoxification pathway becomes ineffective when excess $\mathrm{CN}$ exposure causes endogenous sulfur donors to be exhausted. For this reason, antidotes have been developed to counter the toxicity of CN. Petrikovics et al. [3] recently reviewed CN detoxification methods, available $\mathrm{CN}$ antidotes, and their properties. In the USA, Nithiodote ${ }^{\mathrm{TM}}$ [4] and Cyanokit ${ }^{\circledR}$ [5] are US FDA-approved remedies for $\mathrm{CN}$ intoxication. However, these antidotes require intravenous administration by trained personnel and therefore are not suitable for a mass casualty scenario.

One of the key routes of intervention following $\mathrm{CN}$ exposure is the administration of antidotes that serve as sulfur donors [6, 7]. Thiosulfate is the most important endogenous sulfur donor [8] and is a key component of the 
currently available antidote Nithiodote $^{\mathrm{TM}}$. Dimethyl trisulfide (DMTS) is a novel sulfur donor that has shown superior antidotal potency when compared with thiosulfate in our previous in vitro and in vivo studies [9]. Acute $\mathrm{CN}$ intoxication can be treated with available antidotes [3] such as Nithiodote ${ }^{\mathrm{TM}}$ (comprising a combination of sodium thiosulfate and sodium nitrite) [4] and Cyanokit ${ }^{\circledR}$ (hydroxocobalamin) [5].

DMTS is a naturally occurring oily component of garlic that exhibits poor water solubility of approximately $0.13 \mathrm{mg} / \mathrm{ml}$ [10]. Studies were performed to find formulations with enhanced solubility. The first attempt to formulate this highly lipophilic molecule for intramuscular administration employed polyethylene glycol-2000 (PEG2000)-derived lipid surfactants as micelle-forming agents [10]. However, the $2.5 \mathrm{mg} / \mathrm{ml}$ encapsulation efficiency of the resulting micellar lipids for DMTS was low. A subsequent investigation of additional co-solvents, surfactants, and combinations identified polysorbate 80 (Poly80), also known as Tween 80 , as a promising solubilizing agent for DMTS [11]. Poly80 is a surfactant whose critical micelle concentration (CMC) in water falls in the range of 0.010-0.014 mM [12-14]. Formulation studies showed that $15 \%(\sim 114 \mathrm{mM})$ Poly80 in water elevated DMTS solubility more than 600 times (from approx. 0.13 to $86 \mathrm{mg} / \mathrm{ml})$ [11].

Analytical methods were developed for DMTS detection from formulations, blood, and brain [15, 16]. Methods such as these enabled us to follow the storage stability of DMTS in a $15 \%$ Poly 80 formulation over 29 weeks [17]. By the end of the 29-week study, samples stored at room temperature exhibited 36-58\% reductions in DMTS concentration. The DMTS was stored using a "matroyshka doll" approach in which the DMTS formulation was sealed in a small glass vial with a snap-cap, which itself was placed into a larger vial that was sealed with a crimped septum. Samples stored at $2-8{ }^{\circ} \mathrm{C}$ exhibited a pattern that was statistically indistinguishable from those stored at room temperature. It was hypothesized that upgrading the sealing mechanism might improve the storage stability of formulated DMTS. The goal of the present study was to test whether DMTS formulations that were hermetically firesealed in glass ampules would exhibit better storage stability than formulations stored using the prior snap-cap and septum-sealing approach.

\section{Materials and Methods}

\subsection{Materials}

DMTS, and dimethyl disulfide (DMDS) were purchased from Sigma Aldrich (St. Louis, MO, USA). Acetonitrile (high-performance liquid chromatography [HPLC] grade), water (HPLC), and ethanol were purchased from VWR International (Suwanee, GA, USA). Poly80 was purchased from Alfa Aesar (Haverhill, MA, USA).

\subsection{DMTS Solution Preparation and Storage}

A DMTS stock solution $(50 \mathrm{mg} / \mathrm{ml})$ was prepared by dissolving DMTS in $15 \%$ aqueous Poly 80 . To fully dissolve the DMTS, vigorous hand vortexing was applied for $5 \mathrm{~min}$, followed by $30 \mathrm{~min}$ auto-vortexing at room temperature [18].

\subsubsection{Control Samples (Snap-Cap and Crimp Sealed)}

A set of control samples was prepared by pipetting 1-ml aliquots of DMTS stock solution into 15 snap-cap HPLC vials (VWR International). After capping, each HPLC vial was placed inside a larger 5-ml glass vial (Wheaton, Millville, NJ, USA). Each Wheaton vial was crimp sealed with a rubber septum (Fig. 1). In this manner, 15 samples were prepared and stored at $4{ }^{\circ} \mathrm{C}$ for triplicate testing after 7,14 , 21,60 , and 100 days. On each testing day, the appropriate double-sealed vials were opened and the DMTS concentration was measured by HPLC. Each vial was opened and measured only once. Because we were able to observe clear replication of prior results showing a statistically significant decrease of DMTS within 21 days, the 60- and 100-day samples were not measured.

\subsubsection{Hermetically Sealed Samples}

A hermetically sealed set of samples was prepared by pipetting 1-ml aliquots of DMTS stock solution into 20 ampules ( $2 \mathrm{ml}$; Wheaton). The DMTS content of the solutions was determined via HPLC. The ampules were

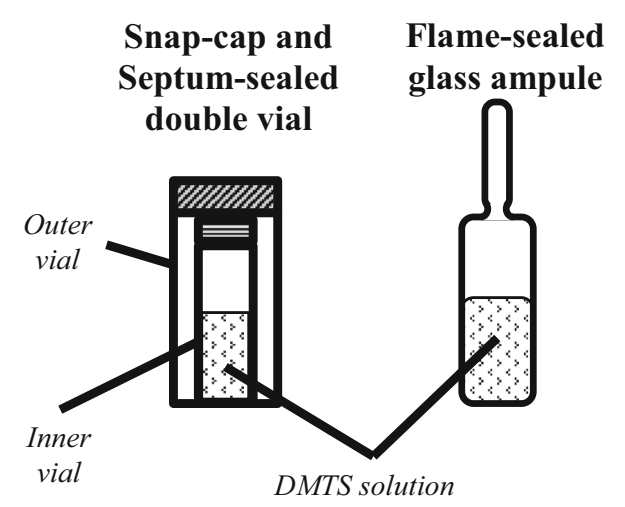

Fig. 1 Two approaches for storing formulated DMTS solution: the snap-cap-sealed vial within a crimp-sealed vial, and the flame-sealed glass ampule 
then manually flame sealed, and stored at $4{ }^{\circ} \mathrm{C}$ for quadruplicate testing by HPLC after 7, 14, 21, 60, and 100 days.

\subsection{HPLC Measurements}

Samples were diluted before HPLC analysis: $990 \mu \mathrm{l}$ of ethanol with $0.1 \mathrm{mg} / \mathrm{ml}$ DMDS (internal standard) was transferred into an HPLC vial; $10 \mu \mathrm{l}$ of DMTS sample solution was then added and the mixture was vortexed well. For the HPLC measurement, the system was a Dionex Ultimate 3000 UHPLC with ultraviolet-visible (UV-VIS) detector (Thermo Scientific). Ten microliters of the diluted sample was injected into a Phenomenex Luna $5 \mu \mathrm{m}$ C8 (2) $100 \mathrm{~A}, 250 \times 4.6 \mathrm{~mm}$ column. The mobile phase consisted of $60 \%$ acetonitrile and $40 \%$ water with a flow rate of $1 \mathrm{ml} / \mathrm{min}$. The detector wavelength was $215 \mathrm{~nm}$. Peak integration was performed by using Chromeleon 7 software. The calibration curve for this assay gave an equation of $y=4.2038 \times-2.0367$ with an $R^{2}=0.9972$.

\subsection{Statistical Analysis}

All data presented are mean \pm standard deviation. Values were compared using analysis of variance (ANOVA) followed by Dunnett's test (GraphPad Prism 5.0, GraphPad
Software Inc., San Diego, CA, USA). An unpaired $t$ test was applied to assess the ampule sealing effect (Fig. 2a). Changes were considered statistically significant at $p<0.05$.

\section{Results}

\subsection{DMTS Stability Studies in Double-Sealed Vials and Ampules}

To allay initial concern that the flame used to hermetically seal the glass ampule samples might itself influence concentrations, we measured the DMTS in six ampules immediately before and after fire sealing the ampules. As seen in Fig. 2a, no significant changes in DMTS concentration were induced by the flame sealing of the ampules. This gave us confidence to proceed with the storage-stability study in the hermetically sealed vials. The DMTS concentrations measured in control samples decreased systematically by $20 \%$ over 21 days (Fig. 2b). In sharp contrast, no statistically significant changes in DMTS concentration (Fig. 2c) were observed over a 100-day period when the DMTS formulation was hermetically stored in fire-sealed ampules.
Fig. 2 Comparing the storage stability of formulated DMTS $(50 \mathrm{mg} / \mathrm{ml}$ in $15 \% \mathrm{w} / \mathrm{w}$ aqueous Poly80 solution): a The effect of ampule flame sealing on DMTS concentration. Statistical analysis for A: unpaired $t$-test, $n=6$. b The DMTS concentration when the formulation was stored in double-sealed vials (snap-capsealed vial within a crimpsealed vial), $n=3$. $\mathbf{c}$ The DMTS concentration when the formulation was stored in flamesealed glass ampules, $n=4$. Data are presented as mean \pm standard deviation. Statistical analysis for (b) and (c): analysis of variance (ANOVA) followed by Dunnett's test; $* * * p<0.001$, all groups were compared with control
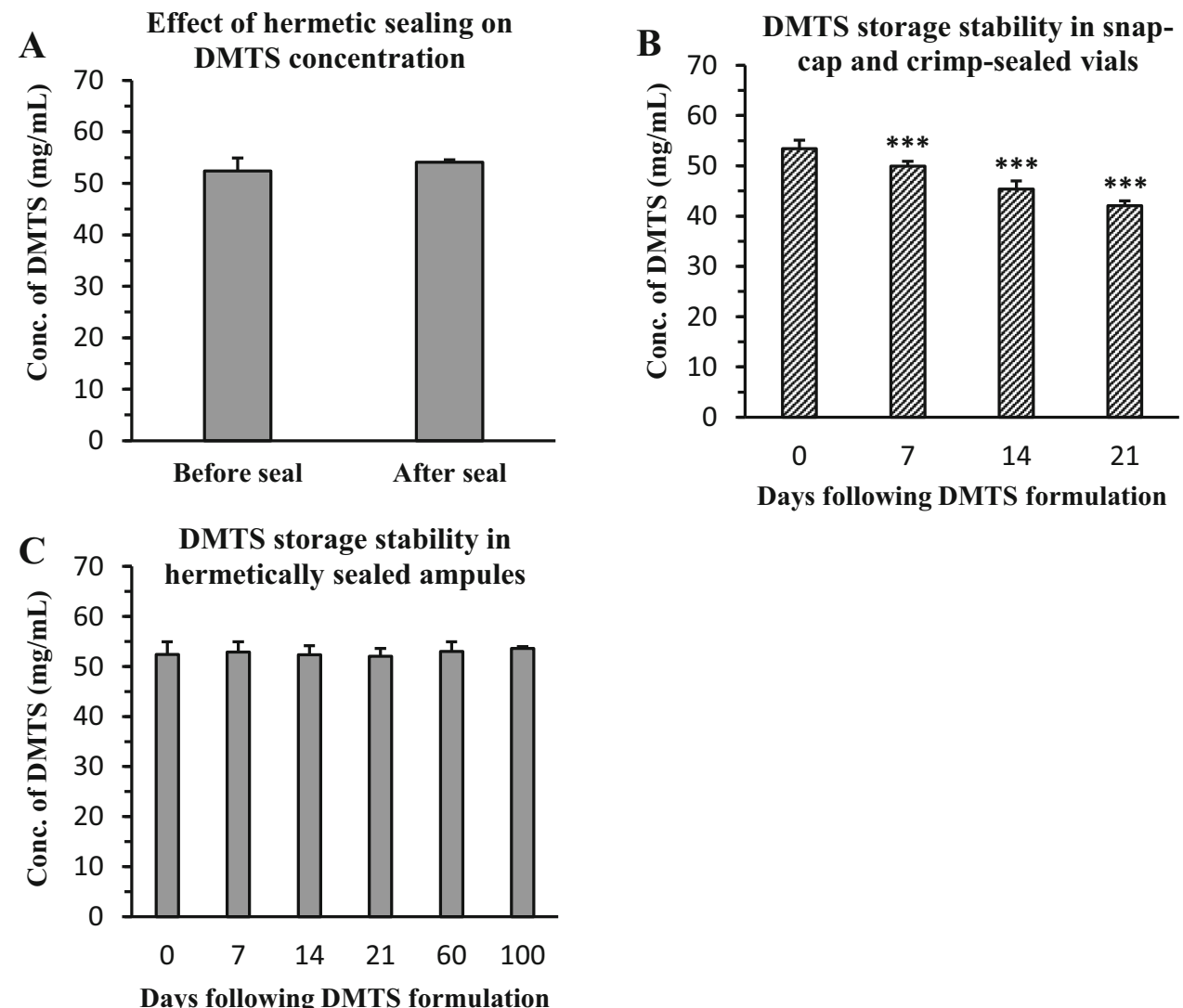

Days following DMTS formulation 


\section{Discussion}

The Poly80 formulation provided a solvent system that enhanced the solubility of the lipophilic DMTS. When the Poly80 concentration exceeds the CMC (in this case by about 600 times), Poly80 forms micelles. Kovacs and colleagues determined the solubility of DMTS to be 0.13 , and $86 \mathrm{mg} / \mathrm{ml}$, respectively, in water [10] and in the $15 \%$ aqueous Poly80 formulation [11]. No changes in DMTS concentration, formulation clarity, or phase were observed upon storage. Based on this assessment, and the encapsulation efficiency $(\mathrm{EE} \%)$ calculation shown below, we estimate that $0.2 \%$ of the DMTS in this formulation was present as free DMTS in the water and $99.8 \%$ was associated with the micelles both before and after storage.

$$
\begin{aligned}
\mathrm{EE} \%= & \frac{\text { amount of encapsulated DMTS }}{\text { total amount of DMTS }} \times 100 \\
= & \frac{(\text { total amount of DMTS }- \text { amount of DMTS in water })}{\text { total amount of DMTS }} \\
& \times 100 .
\end{aligned}
$$

The enhanced solubility enables DMTS to be formulated for intramuscular injections that can be rapidly administered in a scenario of mass intoxication. In a prior study of the storage stability of DMTS in doublesealed containers, Bartling et al. [17] observed DMTS concentration decreased in the range of $7-45 \%$ over 10 weeks of storage at $2-8{ }^{\circ} \mathrm{C}$. They carried out careful experiments attempting to isolate the loss mechanisms. Although these experiments did not lead to a conclusive explanation, the authors suggested that two potential causes of DMTS losses from non-hermetically sealed vials might be reactions with airborne molecules and evaporation. Based on these suggestions, it was hypothesized that higher-quality seals might improve the storage stability of DMTS formulations. The present study tested this hypothesis by replacing snap-cap and crimped-septum seals with hermetic glass seals prepared in a flame-sealing process. The hermetic glass seals did not perturb DMTS concentrations. More importantly, when formulated DMTS was stored at $4{ }^{\circ} \mathrm{C}$ in these hermetically sealed ampules, the storage instability problem was overcome. No losses in DMTS concentration were observed over the 100-day period of this study. Because this hermetic sealing approach shows great promise, studies of DMTS formulation stability over longer storage periods and at different temperatures are ongoing.

\section{Compliance with Ethical Standards}

Conflict of interest This research was supported by the CounterACT Program, National Institutes of Health Office of the Director, and the National Institute of Allergy and Infectious Diseases, NIH/Department of Defense Interagency Agreement (AOD14020-001-00000/
A120-B.P2014-01), and the Robert A. Welch Foundation (X-0011) at Sam Houston State University, Huntsville, TX, USA. L. Kiss, A. Duke, T. Barcza, M. Kiss, and D.E. Thompson have no known conflicts of interest. Dr. Petrikovics [18, 19] is a coauthor of two, and Dr. Kovacs [18] is a coauthor of one, US patents relating to the use of DMTS as a novel cyanide antidote. The authors alone are responsible for the content and writing of the paper.

Open Access This article is distributed under the terms of the Creative Commons Attribution-NonCommercial 4.0 International License (http://creativecommons.org/licenses/by-nc/4.0/), which permits any noncommercial use, distribution, and reproduction in any medium, provided you give appropriate credit to the original author(s) and the source, provide a link to the Creative Commons license, and indicate if changes were made.

\section{References}

1. Petrikovics I, Pei L, McGuinn WD, Cannon EP, Way JL. Encapsulation of rhodanese and organic thiosulfonates by mouse erythrocytes. Fundam Appl Toxicol. 1994;23:70-5.

2. Way JL, Leung P, Cannon E, Morgan R, Tamulinas C, LeongWay J, Baxter L, Nagi A, Chui C. The mechanism of cyanide intoxication and its antagonism. Ciba Found Symp. 1988;140:232-43.

3. Petrikovics I, Budai M, Kovacs K, Thompson DE. Past, present and future of cyanide antagonism research (From the ancient remedies to the recent combination therapy). World J Methodol. 2015;5(2):88-100.

4. Scottsdale AZ. Nithiodote (sodium nitrite injection and sodium thiosulfate injection) Package Insert. Scottsdale: Hope Pharmaceuticals; 2011.

5. Borron SW, Stonerook M, Reid F. Efficacy of hydroxocobalamin for the treatment of acute cyanide poisoning in adult beagle dogs. Clin Toxicol (Phila). 2006;44:5-15.

6. Patterson S, Monteil AR, Cohe JF, Crankshaw DL, Vince R, Nagasawa HT. Cyanide antidotes for mass casualties: watersoluble salts of the dithiane (sulfanegen) from 3-mercaptopyruvate for intramuscular administration. $\mathrm{J}$ Med Chem. 2013;56(3):1346-9.

7. Petrikovics I, Cannon EP, McGuinn WD, Pei L, Way JL. Cyanide antagonism with organic thiosulfonates and carrier red blood cells containing rhodanese. Fundam Appl Toxicol. 1995;24:1-8.

8. Way JL, Cannon EP, Leung P, Davis R, McGuinn DW, Yao C, Chiao C, Nagi A. Antagonism of cyanide intoxication with rhodanese encapsulated within resealed erythrocytes. Adv Biosci. 1991;81:207-11.

9. Rockwood GA, Thompson DE, Petrikovics I. Dimethyl trisulfide: a novel cyanide countermeasure. Toxicol Ind Health. 2016;32:2009-16.

10. Kovacs K, Jayanna PK, Duke A, Winner B, Negrito M, Angalakurthi S, Yu JCC, Füredi P, Ludányi K, Sipos P, Rockwood GA, Petrikovics I. A lipid based formulation for intramuscular administration of a novel sulfur donor for cyanide antagonism. Curr Drug Deliv. 2016;13(8):1351-7.

11. Kovacs K, Duke AC, Shifflet M, Winner B, Lee SA, Rockwood GA, Petrikovics I. Parenteral dosage form development and testing of dimethyl trisulfide, as an antidote candidate to combat cyanide intoxication. Pharm Dev Technol. 2016;7:1-6.

12. Ruiz-Peña M, Oropesa-Nuñez R, Pons T, Louro SRW, PérezGramatges A. Physico-chemical studies of molecular interactions between non-ionic surfactants and bovine serum albumin. Colloids Surf B. 2010;75(1):282-9. 
13. Sigmaaldrich.com. Product information sheet for Tween 80 . Sigma-Aldrich. [online] https://www.sigmaaldrich.com/content/ dam/sigma-aldrich/docs/Sigma-Aldrich/Product_Information_ Sheet/p8074pis.pdf. Accessed 11 Nov. 2017.

14. Chou DK, Krishnamurthy R, Randolph TW, Carpenter JF, Manning MC. Effects of Tween 20 and Tween 80 on the stability of albutropin during agitation. $\mathrm{J}$ Pharm Sci. 2005;94(6):1368-81.

15. Kiss L, Holmes S, Chou CE, Dong X, Ross J, Brown D, Mendenhall B, Coronado V, De Silva D, Rockwood GA, Petrikovics I, Thompson DE. Method development for detecting the novel cyanide antidote dimethyl trisulfide from blood and brain, and its interaction with blood. J Chromatogr B. 2017;1044:149-57.
16. Manandhar E, Maslamani N, Petrikovics I, Rockwood GA, Logue BA. Determination of dimethyl trisulfide in rabbit blood using stir bar sorptive extraction gas chromatography-mass spectrometry. J Chomatogr A. 2016;1461:10-7.

17. Bartling CM, Andre JC, Howland CA, Hester ME, Cafmeyer JT, Kerr A, Petrel T, Petrikovics I, Rockwood A. Stability characterization of a polysorbate 80-dimethyl trisulfide formulation, a cyanide antidote candidate. Drugs R D. 2016;16:109-27.

18. Petrikovics, I, Kovacs, K. Formulations of dimethyl trisulfide for use as a cyanide antidote. US Patent 9,456,996, issued October 4 , 2016.

19. Rockwood GA, Petrikovics I, Baskin SI. Dimethyl trisulfide as a cyanide antidote. US Patent 9,375,407, issued June 28, 2016. 